\title{
A CORRESPONDENCE THEOREM FOR MODULES OVER HOPF ALGEBRAS
}

\author{
JEFFREY BERGEN
}

(Communicated by Lance W. Small)

\begin{abstract}
Let $H$ be a finite-dimensional Hopf algebra. We prove that if $M$ is a faithful $H$-module and if $H_{1} \neq H_{2}$ are sub-Hopf algebras of $H$, then $M^{H_{1}} \neq M^{H_{2}}$, where $M^{H_{1}}$ and $M^{H_{2}}$ are the invariants in $M$ under the respective actions of $H_{1}$ and $H_{2}$. We also show that if $H_{1} \neq H_{2}$, then $H_{1}$ and $\mathrm{H}_{2}$ have different left integrals. Both of these results rely heavily on the freeness theorem of Nichols-Zoeller.
\end{abstract}

In studying the action of a Hopf algebra $H$ on an algebra $A$, one is interested in determining when there exists a Galois correspondence between the sub-Hopf algebras of $H$ and certain subalgebras of $A$. In particular, it is of interest to know when the sub-Hopf algebras of $H$ all have different subalgebras of invariants. Along these lines, the main result of this paper is

Theorem. Let $H$ be a finite-dimensional Hopf algebra with a faithful module $M$. If $H_{1} \neq H_{2}$ are sub-Hopf algebras of $H$, then $M^{H_{1}} \neq M^{H_{2}}$.

Notice that the theorem does not require that $M$ be an algebra or an $H$ module algebra, merely that $M$ be an $H$-module. Some examples of faithful actions of Hopf algebras on algebras are when $A$ is an $H$-module algebra such that the smash product $A \# H$ is simple or when $A$ is a prime $H$-module algebra and $H$ is either the group algebra of a group of "outer" automorphisms or the restricted enveloping algebra of a Lie algebra of "outer" derivations. For further details on smash products, outer automorphisms, and outer derivations, see $[\mathrm{BM}]$.

We now fix the notation we will use throughout this paper. $H$ will denote a finite-dimensional Hopf algebra over a field $k$ with comultiplication $\Delta$, counit $\epsilon$, and antipode $S$. If $K$ is a sub-Hopf algebra of $H$ then the left integral of $K$ will be denoted as $\int_{K}$ and we let $\omega_{K}=K \cap \operatorname{ker} \epsilon$. By a result of LarsonSweedler [LS], $\int_{K}$ is a one-dimensional ideal of $K$ and is the right annihilator in $K$ of $\omega_{K}$. If $M$ is an $H$-module, we let $M^{K}=\{m \in M \mid h \cdot m=\epsilon(h) m$, for all $h \in K\}=\left\{m \in M \mid h \cdot m=0\right.$, for all $\left.h \in \omega_{K}\right\}$ denote the invariants in $M$ under the action of $K$.

Received by the editors September 8, 1992.

1991 Mathematics Subject Classification. Primary 16W30, 16S40.

Research supported in part by NSA Grant MSP-08491. 
If $H_{1}$ and $H_{2}$ are sub-Hopf algebras of $H$, let $K=\left\langle H_{1}, H_{2}\right\rangle$ be the subalgebra of $H$ generated by $H_{1}$ and $H_{2}$. We recall that $\Delta$ and $S$ are, respectively, an algebra homomorphism and an algebra anti-homomorphism. Since $K$ consists solely of sums of products of elements of $H_{1}$ and $H_{2}$, it follows that $\Delta(K) \subseteq K \otimes K$ and $S(K) \subseteq K$. Thus $K$ is also a sub-Hopf algebra of $H$.

Lemma 1. If $M$ is an $H$-module and $H_{1}$ and $H_{2}$ are sub-Hopf algebras of $H$, then $M^{K}=M^{H_{1}} \cap M^{H_{2}}$, where $K=\left\langle H_{1}, H_{2}\right\rangle$.

Proof. Since $K$ contains both $H_{1}$ and $H_{2}$, it is clear that $M^{K}$ is contained in both $M^{H_{1}}$ and $M^{H_{2}}$. Thus $M^{K} \subseteq M^{H_{1}} \cap M^{H_{2}}$. Now suppose $m \in M^{H_{1}} \cap$ $M^{H_{2}}$ and $h=h_{1} h_{2} \cdots h_{n}$, where each $h_{i} \in H_{1} \cup H_{2}$. Since $\epsilon$ is an algebra homomorphism, we have

$$
\begin{aligned}
h \cdot m & =\left(h_{1} h_{2} \cdots h_{n}\right) \cdot m=\left(h_{1} h_{2} \cdots h_{n-1}\right) \cdot\left(h_{n} \cdot m\right) \\
& =\left(h_{1} h_{2} \cdots h_{n-1}\right) \cdot \epsilon\left(h_{n}\right) m=\cdots=\epsilon\left(h_{1}\right) \epsilon\left(h_{2}\right) \cdots \epsilon\left(h_{n}\right) m \\
& =\epsilon\left(h_{1} h_{2} \cdots h_{n}\right) m=\epsilon(h) m .
\end{aligned}
$$

If $h \in K$ then we can write $h=a_{1}+a_{2}+\cdots+a_{s}$, where each $a_{i}$ is a product of elements in $H_{1} \cup H_{2}$. Therefore, our previous argument shows that

$$
\begin{aligned}
h \cdot m & =\left(a_{1}+a_{2}+\cdots+a_{s}\right) \cdot m=a_{1} \cdot m+a_{2} \cdot m+\cdots+a_{s} \cdot m \\
& =\epsilon\left(a_{1}\right) m+\epsilon\left(a_{2}\right) m+\cdots+\epsilon\left(a_{s}\right) m=\epsilon\left(a_{1}+a_{2}+\cdots+a_{s}\right) m=\epsilon(h) m .
\end{aligned}
$$

Thus $m \in M^{K}$ and we have shown the reverse inclusion.

We next use the Nichols-Zoeller [NZ] freeness result to prove a lemma on left integrals.

Lemma 2. If $H$ is a finite-dimensional Hopf algebra and if $H_{1} \neq H$ is a subHopf algebra, then $\int_{H_{1}} \neq \int_{H}$.

Proof. By way of contradiction, suppose there exists some $0 \neq t \in \int_{H_{1}}$ such that $t \in \int_{H}$. The freeness result of Nichols-Zoeller states that $H$ is free both as a left and right $H_{1}$-module. Thus there exists $e_{1}, e_{2}, \ldots, e_{n}$ such that $H=H_{1} e_{1} \oplus$ $H_{1} e_{2} \oplus \cdots \oplus H_{1} e_{n}$. Since the left integral of $H$ is a one-dimensional ideal of $H$, we have $t e_{1}=\lambda_{1} t$ and $t e_{2}=\lambda_{2} t$ for some $\lambda_{1}, \lambda_{2} \in k$. Since $t \in H_{1}$, it follows that both $\lambda_{1}$ and $\lambda_{2}$ are nonzero. Thus $\left(\lambda_{2} t\right) e_{1}-\left(\lambda_{1} t\right) e_{2}=\left(\lambda_{1} \lambda_{2}-\lambda_{1} \lambda_{2}\right) t=0$, contradicting that $e_{1}$ and $e_{2}$ are part of a free basis for $H$ over $H_{1}$. As a result, $\int_{H_{1}} \neq \int_{H}$.

We now combine the previous two lemmas to prove the theorem we mentioned at the outset of this paper.

Theorem 3. Let $H$ be a finite-dimensional Hopf algebra with a faithful module $M$. If $H_{1} \neq H_{2}$ are sub-Hopf algebras of $H$, then $M^{H_{1}} \neq M^{H_{2}}$.

Proof. Suppose not; therefore we will assume that $M^{H_{1}}=M^{H_{2}}$. By Lemma 1, if $K=\left\langle H_{1}, H_{2}\right\rangle$, then $M^{K}=M^{H_{1}}=M^{H_{2}}$. However, if $H_{1} \neq H_{2}$, then $K$ must properly contain either $H_{1}$ or $H_{2}$. Without loss of generality, we may assume that $H_{1} \neq K$, thus we have reduced to the case where $M$ is a faithful $K$-module and $H_{1}$ is a proper sub-Hopf algebra of $K$ such that $K$ and $H_{1}$ have the same set of invariants when acting on $M$.

Let $0 \neq t \in \int_{H_{1}}$; since $\omega_{H_{1}} t=0$ it follows that $\omega_{H_{1}} \cdot(t \cdot M)=0$. Thus $t \cdot M \subseteq M^{H_{1}}=M^{K}$. As a result $\omega_{K} \cdot(t \cdot M) \subseteq \omega_{K} \cdot M^{K}=0$. Since $K$ acts 
faithfully on $M$, we have $\omega_{K} t=0$, and so $t$ belongs to the right annihilator in $K$ of $\omega_{K}$. Hence $t \in \int_{K}$ which contradicts Lemma 2, thereby proving the result.

We can also use Lemmas 1 and 2 to prove

Theorem 4. Let $H$ be a finite-dimensional Hopf algebra. If $H_{1} \neq H_{2}$ are subHopf algebras of $H$ then $\int_{H_{1}} \neq \int_{H_{2}}$.

Proof. We give $H$ the structure of a left $H$-module $M$ by letting $H$ act on $M$ via left multiplication. Note that for any sub-Hopf algebra $J$ we have $M^{J}=\left\{m \in M \mid \omega_{J} \cdot m=0\right\}$, therefore, $M^{J} \cap J=\int_{J}$. By way of contradiction, let $0 \neq t \in \int_{H_{1}}=\int_{H_{2}}$ and let $K=\left\langle H_{1}, H_{2}\right\rangle$. Now, by Lemma 1 , $t \in$ $\left(M^{H_{1}} \cap H_{1}\right) \cap\left(M^{H_{2}} \cap H_{2}\right) \subseteq\left(M^{H_{1}} \cap M^{H_{2}}\right) \cap K=M^{K} \cap K=\int_{K}$. Thus $\int_{K}=$ $\int_{H_{1}}=\int_{H_{2}}$. However, since $K$ must properly contain either $H_{1}$ or $H_{2}$, this contradicts Lemma 2 , thereby proving the result.

In light of the above results, one might suspect that if $K=\left\langle H_{1}, H_{2}\right\rangle$ then $\int_{K}$ is somehow built up from $\int_{H_{1}}$ and $\int_{H_{2}}$. However, this appears not to be the case. For example, suppose $G=S_{n}$, the symmetric group on $n$ letters, and let $g$ be an element of order 2 and $h$ an element of order $n$ such that $g$ and $h$ generate $G$. Let $G_{1}$ and $G_{2}$ be, respectively, the cyclic subgroups of $G$ generated by $g$ and $h$. Given a field $k$, let $H$ be the group algebra $k G$, and let $H_{1}$ and $H_{2}$ be the sub-Hopf algebras $k G_{1}$ and $k G_{2}$. In this case $\int_{H_{1}}$ is the subspace spanned by $1+g$ and $\int_{H_{2}}$ is the subspace spanned by $1+h+\cdots+h^{n-1}$. However, $H=\left\langle H_{1}, H_{2}\right\rangle$ hence $\int_{K}$ is spanned by the sum of all $n$ ! elements of $G$.

\section{REFERENCES}

[BM] J. Bergen and S. Montgomery, Smash products and outer derivations, Israel J. Math. 53 (1986), 321-345.

[LS] R. G. Larson and M. E. Sweedler, An associative bilinear form for Hopf algebras, Amer. J. Math. 91 (1969), 75-94.

[NZ] W. D. Nichols and M. Bettina Zoeller, A Hopf algebra freeness theorem, Amer. J. Math. 111 (1989), 381-385.

Department of Mathematics, DePaul University, Chicago, Illinois 60614

E-mail address: mat jmb@depaul. bitnet 